\title{
Bei der Diätberatung kommen dicke Ärzte besser an
}

Schenken übergewichtige Patienten ihrem Hausarzt mehr Vertrauen, wenn dieser ebenfalls mit den Kilos zu kämpfen hat? Einer US-amerikanischen Studie zufolge werden zumindest Diätempfehlungen von korpulenten Ärzten besser angenommen.

In ihrer Längsschnittstudie testeten die Autoren um Dr. Sara N. Bleich und Kollegen von der Johns Hopkins Bloomberg School of Public Health in Baltimore zwei Variablen: Vertrauen dem Arzt gegenüber ganz allgemein und Vertrauen im Hinblick auf dessen Fähigkeit, die Patienten beim Abspecken zu unterstützen. Dazu wurden 600 übergewichtige Teilnehmer (BMI $\geq 25 \mathrm{~kg} / \mathrm{m}^{2}$ ) via Internet befragt. Alle hatten im Vorjahr mindestens einmal eine Hausarztpraxis aufgesucht.

Das allgemeine Vertrauen, das die Teilnehmer ihrem Arzt entgegenbrachten, war insgesamt hoch, unabhängig vom BMI des Doktors. Deutliche Unterschiede gab es dagegen beim Vertrauen in die ärztliche Diätberatung: Je mehr Pfunde die Ärzte auf die Waage brachten, desto besser schnitten sie hier in den Augen ihrer Patienten ab. Die Wahrscheinlichkeit, dass ein Patient in dieser Hinsicht seinem Arzt vertraute, lag bei $87 \%$ bzw. $82 \%$ für übergewichtige bzw. adipöse

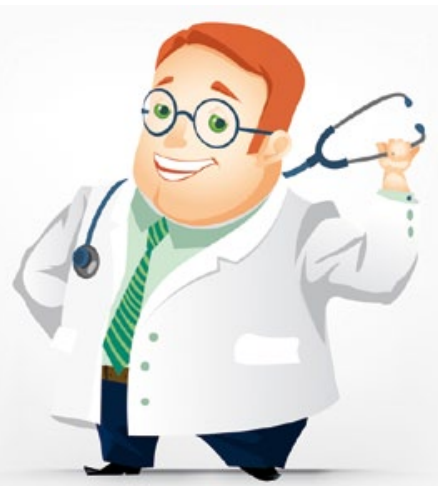

Ärzte, aber nur bei 77\% für Ärzte ohne Gewichtsprobleme. Bei den ärztlichen Ratschlägen zu Bewegungsmaßnahmen und Gewichtskontrolle stieg das Vertrauen der Patienten im Trend ebenfalls mit dem ärztlichen BMI, die Unterschiede waren hier jedoch nicht signifikant.

Vor allem stark übergewichtige Patienten scheuen aus Scham oder Angst vor negativen Reaktionen häufig den Arztbesuch. Nach Bleich und Kollegen ist das offenbar nicht ganz unbegründet. So hätten frühere Studien gezeigt, dass adipöse Patienten vom Arzt schlechter behandelt würden, dieser weniger Zeit für sie aufwende und bestimmte Untersuchungen weniger bereitwillig durchführe.

EO -

Bleich SN et al. How does physician BMI impact patient trust and perceived stigma? Preventive Medicine 2013; in press

\section{Haar oder Herz : Der Körper setzt Prioritäten}

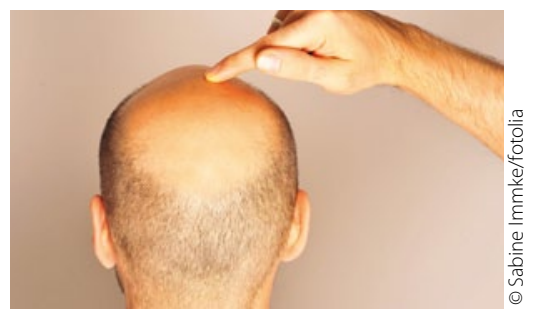

Infarktalarm?

Die kausale Verknüpfung von Erkrankungen anatomisch weit auseinander liegender Organe ist zu einem beliebten Hobby der Wissenschaftler geworden. So hatte man schon vor einigen Jahren zeigen können, dass der fehlende Blinddarm das Risiko für einen Herzinfarkt deutlich erhöht. Die Erklärung ist einfach: Steht der Blinddarm nicht mehr zur Verfügung, muss sich der Organismus andere Ziele suchen, um sein Entzündungsbedürfnis ausleben zu können.
Und was liegt da näher als die Koronararterien?

Auch in einer anderen Hinsicht steht der Magen-Darm-Trakt mit dem Herzen in Zusammenhang. So sagt der bayerische Volksmund: "Wenn's Arscherl brummt, ist's Herzerl g'sund." Wer möchte dem wiedersprechen, zumal man im klinischen Alltag immer wieder erlebt, dass hinter einem vermeintlichen akuten Koronarsyndrom doch nur ein eingeklemmter Furz steckt.

Sehr interessant ist eine aktuelle Studie, die den Kopf mit dem Herzen in Verbindung bringt. Die neue Erkenntnis lautet: Männer mit Glatze sind besonders infarktgefährdet, aber nur dann, wenn sich die lichte Stelle am Hinterkopf zeigt. Männer mit frontaler Haarlosigkeit, also solche, die mehr Platz brauchen für ihr schönes Gesicht, sind nicht betroffen.
Und wie es bei einer Vollglatze ist, weiß niemand so genau.

Nun stellt sich natürlich die Frage, ob man da etwas präventiv machen sollte, ob also eine das Haarwachstum fördernde Behandlung oder gar eine Haartransplantation à la Berlusconi das kardiovaskuläre Risiko günstig beeinflussen kann. Eine solche Interventionsstudie wäre sicherlich wünschenswert. Aber wie lässt sich das Zusammenspiel zwischen Ursache und Wirkung erklären? Unbestritten benötigen KHK-Patienten vermehrt Wachstumsfaktoren, um neue Gefäße bilden zu können. Und wenn solche am Herzen dringend gebraucht werden, bleibt für den Kopf, also das Haarwachstum nichts mehr übrig. Der Körper setzt eben Prioritäten. Oder fällt Ihnen etwas noch Unsinnigeres ein? Dr. Peter Stiefelhagen . 